\title{
Critical attenuation of sound of liquid helium in restricted geometries
}

\author{
B. Lambert, R. Perzynski and D. Salin \\ Laboratoire d'Ultrasons (*), Université Pierre-et-Marie-Curie, Tour 13, 4 place Jussieu, 75230 Paris Cedex 05, France
}

(Reçu le 4 octobre 1979, révisé le 9 novembre 1979, accepté le 19 novembre 1979)

\begin{abstract}
Résumé. - Nous avons mesuré l'atténuation critique ultrasonore de l'hélium quatre liquide confiné dans du verre poreux de Vycor. Près de $T_{\lambda}$, les résultats expérimentaux sont interprétés en terme de loi d'échelle en géométrie réduite. Ces résultats sont comparés à ceux obtenus pour l'hélium dans différentes géométries réduites.
\end{abstract}

\begin{abstract}
We have measured the critical attenuation of sound of liquid helium four confined in porous Vycor glass. Around $T_{\lambda}$ the experimental data are interpreted in terms of restricted geometry scaling. We compare our results with other results obtained for $\mathrm{He}^{4}$ in different restricted geometries.
\end{abstract}

1. Introduction. - In the last years there has been a great interest in the study of the so-called restricted geometries such as superconductor films or superfluid films. In such particular cases the size of the system, i.e. film thickness or pore radius, can be smaller than some characteristic lengths of the bulk and then their properties are modified. In superfluid helium four, around $T_{\lambda}$, the normal-superfluid transition temperature, the coherence length $\xi$ is the only relevant variable of the transition; when the size of the system is smaller than $\xi$, the transition by itself will be modified.

The purpose of this paper is the study of the sound attenuation $\alpha$ of liquid helium confined in restricted geometries. Around $T_{\lambda}$, the measurement of this transport coefficient $(\alpha)$ is a test of the critical dynamics of the transition.

In a first step we will recall the theoretical predictions of scaling in restricted geometries. We then describe our experimental set-up and analyse our results on helium confined in porous Vycor glass. As an extension of this experiment we compare our results with other results obtained in other restricted geometries such as helium films.

2. Scaling of confined helium near $\boldsymbol{T}_{\lambda}$. - For bulk $\mathrm{He}^{4}$ static scaling arguments are used to explain critical properties such as specific heat [1]. The basis of the scaling hypothesis consists in emphasizing the importance of the correlation length $\xi(T)$.

(*) Associated with the Centre National de la Recherche Scientifique.
For the sound attenuation which is of our experimental interest, using dynamic scaling ideas and mode-mode coupling calculations, Kawasaki [2] shows that the contribution due to the fluctuations of the order parameter can be expressed as a function of $\omega \tau_{\mathrm{F}}:$

$$
\alpha / \alpha_{\lambda}=f\left(\omega \tau_{\mathrm{F}}\right)
$$

where $\tau_{F}$ is a characteristic time of the fluctuations. From dynamic scaling one would expect :

$\tau_{\mathrm{F}} \propto \xi^{z} \propto \varepsilon^{-v z}$ with $v=2 / 3$ and $z \simeq 3 / 2[3] ;$

$\varepsilon$ is the reduced temperature :

$$
\varepsilon=\frac{\left|T-T_{\lambda}(\infty)\right|}{T_{\lambda}(\infty)}
$$

the attenuation at $T=T_{\lambda}(\infty)$ noted $\alpha_{\lambda}$ varies with the frequency $\omega / 2 \pi$ [2]. Note that this contribution to the attenuation is predominant in experiments on bulk helium performed at $\mathrm{GHz}$ frequencies and the accord of the scaling functions with experimental data is quite good [4].

For $\mathrm{He}^{4}$ in restricted geometry, Fisher [5] has given the form of the static scaling law. The superfluid transition is obtained when the correlation length reaches the magnitude of a characteristic dimension $d$ of the finite system (or the thickness of a film), say $\xi(\varepsilon) \sim d$. The only relevant variable affecting the change over from bulk to finite system is the ratio $\xi(\varepsilon) / d$. The accordance with experimental results is quite satisfactory $[6,7]$.

Using renormalization group theory Suzuki [8] confirms Fisher's results and extends them to the 
dynamic case. This dynamic scaling law yields two types of cross-over effects corresponding to the size $(d)$ and time $(t)$. For sound attenuation, if we emphasize that the main contribution is due to a fluctuation mechanism we can write it as :

$$
\alpha \propto f^{\prime}\left(d^{0} \varepsilon, d^{-z} t\right)
$$

with $\theta=1 / v$. This is reduced to the static scaling function $f^{\prime}\left(d^{0} \varepsilon\right)$ if the time dependence of eq. (2) is neglected.

3. Experiment. - The Vycor glass [9] we use is an array of multiconnected tiny diameter pores. We have determined the porosity and the effective surface of our samples by the so-called B.E.T. method [10]. We find that there are two types of sample (respectively $V_{1}$ and $V_{2}$ ); both of them have the same porosity $x_{1}=x_{2}=(31 \pm 1 \%)$ in volume but the effective surfaces are different : $s_{2}=280 \mathrm{~m}^{2} / \mathrm{cm}^{3}$ and $s_{1}=160 \mathrm{~m}^{2} / \mathrm{cm}^{3}$. The diameters of the pores are then estimated :

$$
2 R_{2}=(75 \pm 5) \AA \quad \text { and } \quad 2 R_{1}=(130 \pm 10) \AA .
$$

Before measuring the acoustical attenuation of helium confined in such a substrate, we have to measure the acoustical property of empty Vycor. We use a classical transmission method through a sample of Vycor of typical thickness of $4 \mathrm{~mm}$; the transducers are made of $\mathrm{ZnO}$ deposited on Platinum ; we are able to work with such a device from $0.5 \mathrm{GHz}$ to $3 \mathrm{GHz}$. The sample is in a cell which is immersed in a helium bath.

We have measured the acoustical attenuation $\alpha_{\mathbf{v}}$ of the empty Vycor in the frequency range $0.5 \mathrm{GHz}$ $2 \mathrm{GHz}$ and for temperature $T$ from $80 \mathrm{mK}$ to $2.5 \mathrm{~K}$ [11]. A fit to the data gives the attenuation

$$
\alpha_{\mathrm{v}}=A \omega^{0} T^{3}
$$

with

$$
A_{1}=2.25 \mathrm{~dB} \mathrm{~cm}^{-1} \mathrm{~K}^{-3}
$$

and

$$
A_{2}=3.10 \mathrm{~dB} \mathrm{~cm}^{-1} \mathrm{~K}^{-3}
$$

for the two types of Vycor. The experimental data for Vycor 2 are shown in figure $1\left(\alpha_{\mathrm{V}}\right)$. Such frequency and temperature dependences are observed for amorphous compounds in a high power regime where the resonant attenuation is completely saturated [12]; the values of $A$ are of the same order as for silica which is $96 \%$ of the Vycor glass.

Now we have to measure the attenuation of Vycor full of helium. The attenuation of the empty Vycor being independent of frequency our experimental frequency range must satisfy two conditions : (i) the frequency must be high enough in order that the helium attenuates more than the Vycor; (ii) the frequency must not be too high in order to maintain a sufficient signal from the helium. We have found a window between $500 \mathrm{MHz}$ and $1.100 \mathrm{MHz}$; this

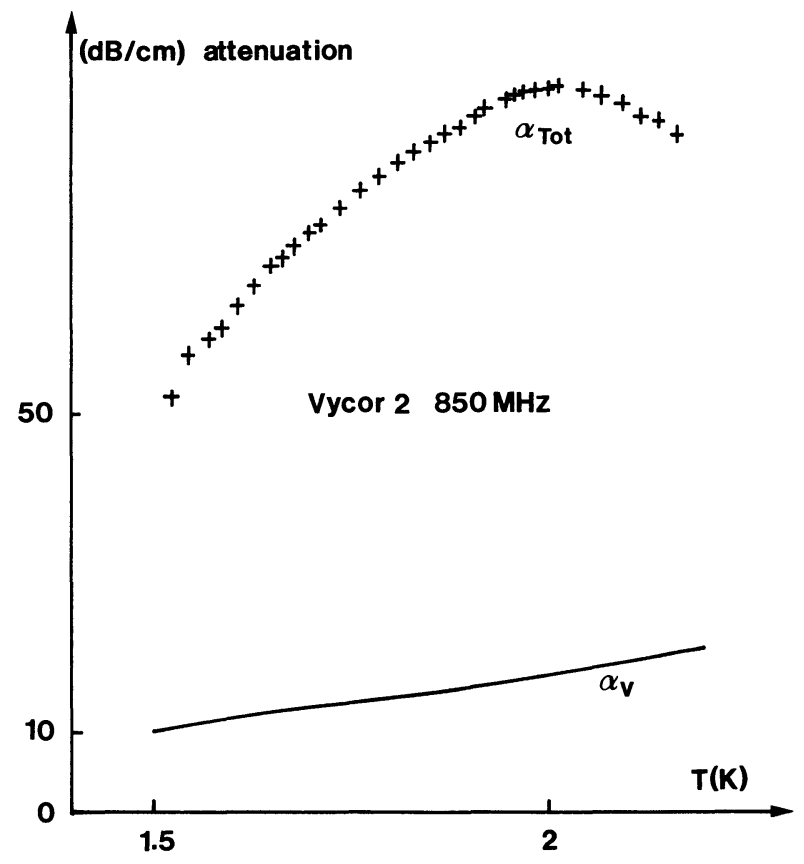

Fig. 1. - Ultrasonic attenuation of empty Vycor (full line) and of Vycor full of helium (crosses).

frequency range is interesting because recent experimental results exist in bulk liquid helium around $T_{\lambda}$ [4].

We have now to analyse our data for Vycor full of helium; a first simple analysis would then be to substract the attenuation $\alpha_{\mathrm{v}}$ of the empty Vycor from the total attenuation $\left(\alpha_{\text {Tot }}\right)$. Such an analysis ignores the fact that the sound wave propagates through a medium made of two compounds (vycor and helium). Such a problem has not received, to our knowledge, a solution; anyway in our present case we are mainly interested in the reduced from $\alpha_{c} / \alpha_{c m}$ where $\alpha_{c m}$ is the critical attenuation at the maximum. The use of this reduced form added to the fact that $\alpha_{\text {Tot }} \gg \alpha_{\mathbf{v}}$ (see Fig. 1) allow us to ignore the details of the propagation. Around $T_{\lambda}$ the total attenuation exhibits a maximum for $T \sim 2 \mathrm{~K}$. For temperature above this maximum, $\alpha_{\text {Tot }}$ decreases as $\alpha_{\mathrm{V}}$ increases, and a detailed knowledge of the propagation would be needed. For this reason, we only analyse our data for temperatures below this maximum.

4. Experimental results. - The attenuation $\alpha_{\mathrm{He}}$ is the sum of a critical $\alpha_{c}$ and a non-critical $\alpha_{B}$ contribution. The estimation of $\alpha_{B}$ for $T<T_{\lambda}$ is complicated even in bulk case [4] : we have to take into account not only the contribution of the shear viscosity but also that of elementary excitations (phonons and rotons). In the case of helium in restricted geometry we can say nothing about this last contribution. So we take for $\alpha_{B}$ an extrapolation of the variation of attenuation we have observed between $1 \mathrm{~K}$ and $1.5 \mathrm{~K}$ [11] : this background correspond at $850 \mathrm{MHz}$ to a variation of about of $15 \mathrm{~dB} \mathrm{~cm}^{-1} \mathrm{~K}^{-1}$ for the two types of Vycor. 
The maximum of the attenuation $\alpha_{c}$ corresponds to a shift in temperature

$$
\Delta T=T_{\lambda}(\infty)-T_{\mathrm{m}}(R), \Delta T_{1}=70 \mathrm{mK}
$$

for Vycor $1\left(2 R_{1}=130 \AA\right)$ and of $\Delta T_{2}=200 \mathrm{mK}$ for Vycor $2\left(2 R_{2}=75 \AA\right)$; this shift is independent of the frequency.

5. Discussion. - If we assume that the temperature of the maximum $T_{\mathrm{m}}$ is the temperature $T_{\lambda}$ where the transition occurs, we have now to compare the experimental shifts in temperature of the transition with Fisher's prediction :

$$
\Delta T_{1} / \Delta T_{2}=\left(d_{2} / d_{1}\right)^{\theta}
$$

where $d_{1}$ and $d_{2}$ are the thickness of superfluid helium in the two types of Vycor; this means that $d_{\mathrm{i}}$ must included neither the solid layer close to the substrate, nor the non-superfluid one [13, 14]. In a recent article [15] we have proposed a length $\delta_{0}(T)$ as a measure of the thickness of non-superfluid helium. We write $d_{\mathrm{i}}=2\left[R_{\mathrm{i}}-\delta_{0}(T)\right]$. In our case

$$
\Delta T=T_{\lambda}(\infty)-T \geqslant 70 \mathrm{mK}
$$

we get $\delta_{0} \simeq 10 \AA$, quite temperature independent. The value $\theta$ we deduce from eq. (4) is $\theta=1.52$ in agreement with theoretical predictions [16]; we will suggest that this procedure will reconcile previous experiments [6] with the theory. The value of $\delta_{0}$ we use is a little larger compared to the thickness of solid helium ( $\sim 8 \AA$ ) [13] which leads to $\theta=1.56$; but $\delta_{0}$ includes also the non-superfluid part of the liquid helium of the film.

We have to notice that an expression similar to (4) may also be obtained from Ginzburg-Pitaevskii calculations [17]; moreover these authors show that the transition occurs in a helium film (thickness $d$ ) at a temperature shifted from $T_{\lambda}$ in order to fulfil the condition

$$
d / 2=(\pi / 2) \xi
$$

These calculations give in other geometries [18] :

$$
\begin{aligned}
& \text { cylinder of radius } R: R=2.4 \xi \\
& \text { sphere of radius } R_{\mathrm{s}}: R_{\mathrm{s}}=\pi \xi .
\end{aligned}
$$

Using these results it is interesting to discuss an experimental result of Chen and Gasparini [6] : these authors measure the temperature of the specific heat maximum for regular cylinder (Nuclepore filters) full of helium and for thin films. To fit the data with the same parameter for both film and filled channel, they have to take an equivalent film thickness 0.585 times the diameter of the cylinder. This numerical value has to be compared to the ratio $(\rho / 2) / 2.4=0.65$ between film (5) and cylinder (6).

In figure 2 we compare the experimental results for the two types of Vycor with the scaling function

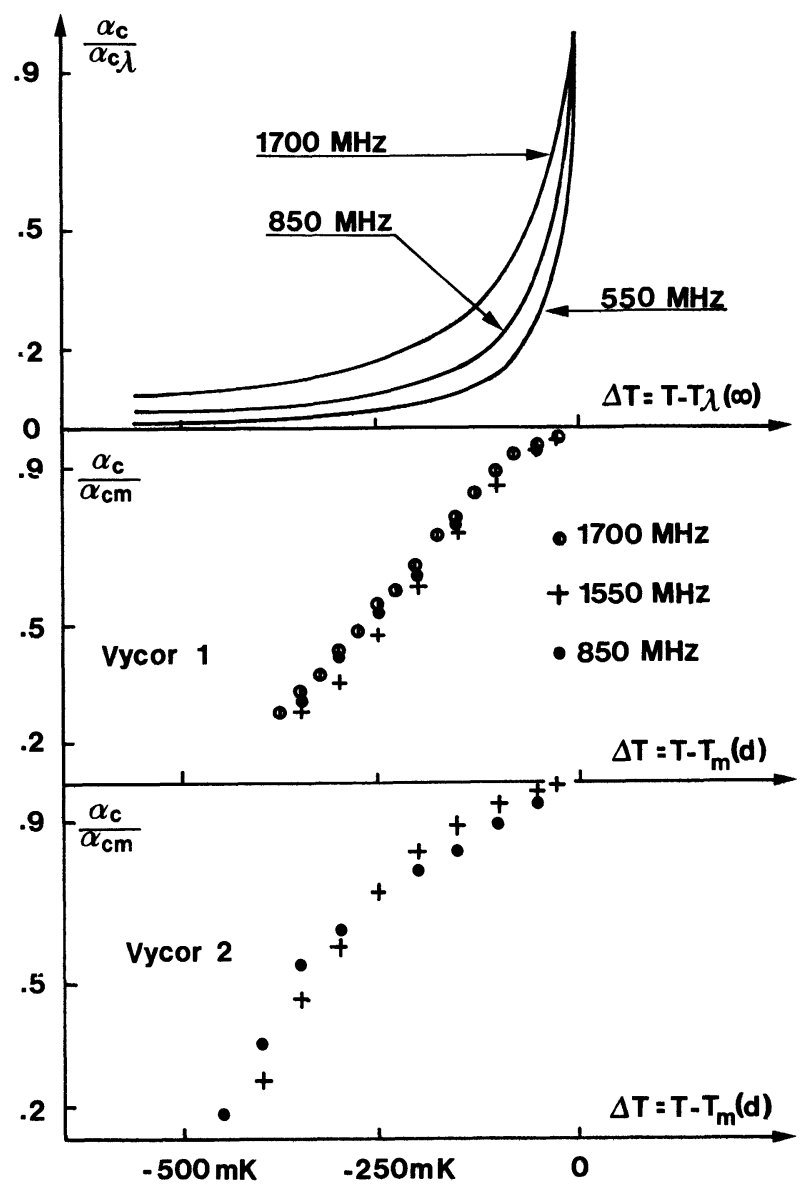

Fig. 2. - Relative attenuation versus $\Delta T$, with $\Delta T=T-T_{\lambda}(\infty)$ for bulk $\mathrm{He}^{4}$ and $\Delta T=T-T_{\mathrm{m}}\left(d_{\mathrm{i}}\right)$ for $\mathrm{He}^{4}$ confined in Vycor i.

which fits the experimental bulk data [4]. For bulk $\mathrm{He}^{4}$ we use $\Delta T=T_{\lambda}(\infty)-T$ and plot the relative value $\alpha_{c} / \alpha_{c_{\lambda}}$ in order to compare with the scaling function $f\left(\omega \tau_{\mathrm{F}}\right)$. For $\mathrm{He}^{4}$ confined in restricted geometry we plot versus $\Delta T=T_{\mathrm{m}}-T$, the ratio $\alpha_{\mathrm{c}} / \alpha_{\mathrm{cm}}$ with $\alpha_{\mathrm{cm}}$ the value of the critical attenuation for $T_{\mathrm{m}}(d)$. In opposition to the bulk case we see that for a given Vycor sample the values of $\alpha_{\mathrm{c}} / \alpha_{\mathrm{cm}}$ are the same for different frequencies. This fact and more significatively the frequency independent position of the maximum indicates that we are in the static case. Finally we plot the values $\alpha_{\mathrm{c}} / \alpha_{\mathrm{cm}}$ versus $d^{\theta} \varepsilon$ in order to find a static scaling function : with the values $\theta$ and $d_{i}$ quoted before, it was not possible to scale the experimental data on the same curve. Such an impossibility for Vycor data has already been reported for heat capacity measurements [19]; Does this mean that such a geometry is not well defined ? It would be interesting to have a much better defined geometry such as plane $\mathrm{He}^{4}$ film.

In order to test the idea of scaling function in restricted geometries, we reanalyse our previous experimental data on the attenuation of sound at $9 \mathrm{GHz}$ in plane $\mathrm{He}^{4}$ films deposited on quartz [20]. In such an experiment the substrate is much more well defined than the Vycor. We analyse the critical 
attenuation measured for two film thicknesses : $D_{1}=81 \pm 3 \AA$ and $D_{2}=207 \pm 3 \AA$ [13]. The superfluid thicknesses, $d_{\mathrm{i}}=D_{\mathrm{i}}-\delta_{0}$, are obtained as explained above [15]. For these films the critical attenuation exhibits a plateau close to the transition $\left(\alpha_{\mathrm{cp}}\left(d_{\mathrm{i}}\right)\right)$. In figure 3 , we plot the ratio $\alpha_{\mathrm{c}} / \alpha_{\mathrm{cp}}$ versus $d^{\theta} \varepsilon\left(\alpha_{\mathrm{cp}}\left(d_{2}\right) / \alpha_{\mathrm{cp}}\left(d_{1}\right)=3.3\right)$; in this case (which follows the general shape of the heat capacity data [21]) we obtain a levelling off (due to the plateau) rather than a rounding as for Vycor. Figure 3 clearly shows that for these films, the data are well scaled by a function of $d^{\theta} \varepsilon$. We have to notice that in this very high frequency regime the attenuation of sound is explained by interaction via first sound modes with a characteristic time $\tau_{1} \sim \xi / c_{1}\left(c_{1}\right.$ first sound velocity); this time is clearly different from $\tau_{\mathrm{F}}$ which corresponds to the interactions of sound via second sound modes.

6. Conclusion. - We have measured the critical ultrasonic attenuation of helium confined in restricted geometries (Vycor and helium films). The general outline of the experimental data are explained in the

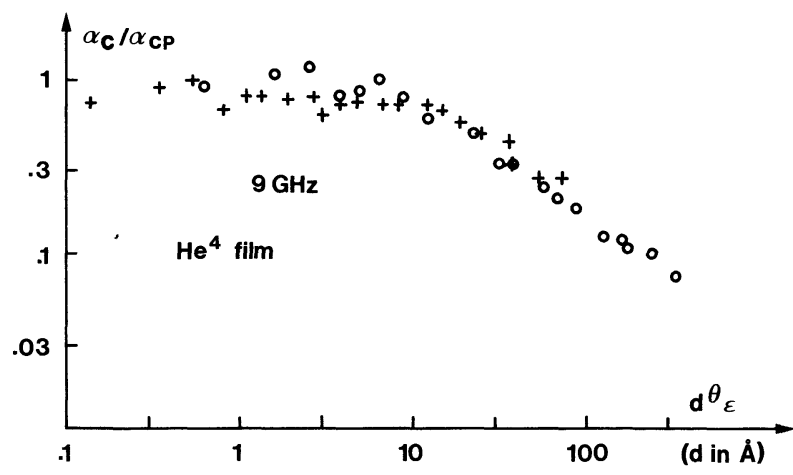

Fig. 3. - Scaling plot of the relative attenuation versus $d^{\theta} \varepsilon$ with $\theta=1 / v=1.5$ and $\varepsilon=\left(T_{\lambda}(\infty)-T\right) / T_{\lambda}(\infty)$.

framework of scaling in restricted geometries. In the case of Vycor the scaling is frequency independent but we are not able to find an unique scaling function for the two types of Vycor. For plane $\mathrm{He}^{4}$ film, using previous experimental data, we are able to find the static scaling function in agreement with Fisher's predictions.

\section{References}

[1] A review of these properties is given by AHLERS, G., in The Physics of Liquid and Solid Helium, Edited by K. H. Bennemann and J. B. Ketterson (J. Wiley, New York), 1976, Part I, Chap. 2.

[2] Kawasaki, K., Int. J. Magn. 1 (1971) 171.

[3] Hohenberg, P. C. and Halperin, B. I., Rev. Mod. Phys. 49 (1977) 435, Sect. VI B.

[4] Lambert, B., Perzynski, R. and Salin, D., J. Physique Colloq. 39 (1978) C6-148; Phys. Rev. B 19 (1979) 1025; J. Physique Lett. 40 (1979) L-477.

[5] Fisher, M. E., in Critical Phenomena, Proceedings of the International School of Physics "Enrico Fermi", Course 51, edited by M. S. Green (Academic Press, New York), 1971.

[6] Chen, T. and Gasparin, F. M., Phys. Rev. Lett. 40 (1978) 331.

[7] Wiechert, H. and Wupperfield, G., J. Physique Colloq. 39 (1979) C6-324 and private communication.

[8] Suzuki, M., Prog. Theor. Phys. 58 (1977) 1142.

[9] The porous Vycor glass (No 7930) used in the present experiment was obtained from Corning Glass (U.S.A.)

[10] Young, D. M. and Crowell, A. D., Physical Adsorption of Gases (Butterworths, London), 1962.

[11] The low temperature results are in : SALIN, D., Thesis (Université Pierre-et-Marie-Curie, Paris, 1979); they will be published in a forthcoming paper.
[12] Hunklinger, S. and Arnold, W., in Physical Acoustics, edited by R. N. Thurston and W. P. Mason (Academic Press, New York), 1976, Vol. 12, p. 155.

[13] Lambert, B., Perzynski, R., Salin, D. and Joffrin, J., J. Low Temp. Phys. 28 (1977) 359.

[14] Oestereich, T. and Stenschke, H., Phys. Rev. B 16 (1977) 1996.

[15] Lambert, B., Levelut, A., Perzynski, R. and Salin, D., J. Low Temp. Phys. 37 (1979) 679.

[16] Bray, A. J. and Moore, M. A., J. Phys. A : Math. Gen. 11 (1978) 715.

[17] Ginzburg, V. L. and Pitaevskil, Zh. Eksp. Theor. Fiz. 34 (1958) 1240 ; Sov. Phys. J.E.T.P. 34 (1958) 858.

[18] Mamaladze, Yu. G., Sov. Phys. J.E.T.P. 52 (1967) 479.

[19] Joseph, R. A. and Gasparini, F. M., J. Physique Colloq. 39 (1978) C6-310.

[20] Joffrin, J., Lambert, B. and Salin, D., J. Physique Lett. 37 (1976) L-255.

[21] Brewer, D. F., in The Physics of Liquid and Solid Helium, Part II, edited by K. H. Bennemann and J. B. Ketterson (J. Wiley and sons, New York), 1978, chap. 6 : Multilayer adsorbed helium in restricted geometries. 